\title{
Es geht vorwärts mit der E-Card
}

\author{
Im Projekt elektronische Gesund- \\ heitskarte ist eine Entscheidung \\ zur Finanzierung der Lesegeräte \\ gefallen. Damit steht für Ärzte nun \\ fest, dass sie zumindest auf diesen \\ Kosten nicht sitzen bleiben.
}

— Eigentlich sollten die Patienten schon seit 2006 ihre neuen Gesundheitskarten in deutschen Arztpraxen zücken. In den vergangenen Jahren ist das Projekt elektronische Gesundheitskarte (eGK) aber immer wieder ins Stocken geraten. Nun haben sich Ende Oktober nach langem Streit der Spitzenverband der gesetzlichen Krankenversicherung und die Kassenärztliche Bundesvereinigung (KBV) über die Finanzierung der technischen Ausstattung für niedergelassene Ärzte geeinigt: Demnach sollen niedergelassene Ärzte für die Anschaffung eines stationären Lesegeräts 355 Euro erhalten und für mobile Geräte 280 Euro. Für die Installation soll es 215 Euro geben.
Die Beträge sollen so berechnet sein, dass Ärzte dafür auf jeden Fall ein Kartenlesegerät bekommen. Wer ein technisch anspruchsvolleres Gerät möchte, muss drauflegen. Die Einigung kommt der Industrie entgegen, die seit Jahren die Entwicklungen vorfinanziert.

\section{Kartenausgabe noch schleppend}

In Sachen Kartenausgabe, also beim "Rollout" hat sich bislang kaum etwas bewegt. Dabei gibt es auch Lösungen für Ärzte, die nicht mit ihrer kompletten Praxis-EDV online gehen wollen. Möglich wäre laut Dr. Pablo Mentzinis, eGK-Experte beim Herstellerverband BITKOM, dass der Kartenleser die Technik für den Online-Gang enthält oder dass er von der Praxis-EDV abgekapselt wird und über den Konnektor, der eine sichere Verbindung zur Telematik-Infrastruktur bzw. zu den Krankenkassen herstellt, online geht. Richtig weitergehen wird es mit dem Rollout der eGK, der in der Startregion

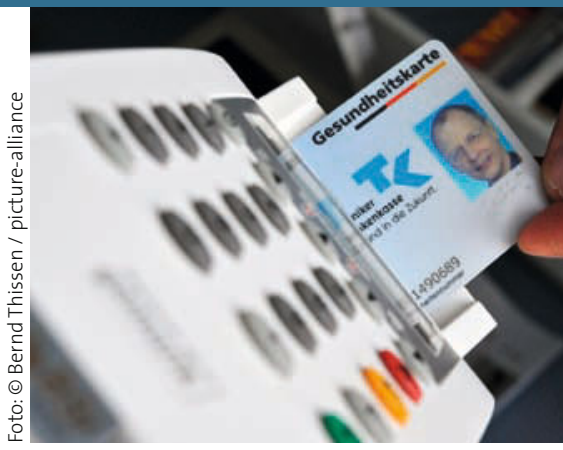

Doch keine unendliche Geschichte?

Nordrhein nur schleppend angelaufen ist, wohl erst 2011.

\section{Bundesregierung macht jetzt Druck}

Als nächste Etappe im Basisrollout stehen nach Nordrhein Westfalen-Lippe, Niedersachsen, Bremen und Hamburg auf der Agenda. Bislang sind die Kassen mehr als zurückhaltend mit dem Thema umgegangen. Die Bundesregierung macht aber jetzt Druck: Durch eine Änderung im GKV-Finanzierungsgesetz verpflichtet sie die Krankenkassen, bis Ende 2011 an mindestens 10\% ihrer Versicherten eGK auszugeben. Tun sie das nicht, sollen die Verwaltungsausgaben der Kassen 2012 gegenüber 2010 um 2\% gekürzt werden.

ILSE SCHLINGENSIEPEN/RebeKKA HöHL -

\section{Meldungen}

\section{Erster UMTS-Router fürs KV-SafeNet zertifiziert}

Der KV-SafeNet-Provider telemed hat mit dem Lancom 1751 UMTS nach eigenen Angaben erstmals einen UMTS-VPN-Router für die Anbindung niedergelassener Ärzte an die KVen zertifiziert. Damit könnten Ärzte ohne DSL-Zugang, etwa auf dem Land, wo teilweise noch keine flächendeckende DSL-Anbindung vorhanden ist, ihre Abrechnungen online über telemed tätigen. Der Lancom 1751 UMTS ist der vierte Lancom Router, der fürs KV-SafeNet zertifiziert wurde. www.telemed.de

\section{GUSbox ${ }^{\circledast}$ erweitert das Portfolio von dgnservice}

Mediamed Systec und dgnservice haben eine Partnerschaft bei Endgeräten vereinbart. Die gemeinsamen Leistungen sollen von Online-Datensicherung über rechtssichere elektronische
Dokumentation und Archivierung bis hin zum elektronischem Arztbrief mit qualifizierter Signatur reichen. Ab sofort vervollständigt die von der Gemeinschaft unabhängiger Softwarehäuser (GUS) und Mediamed Systec entwickelte Kommunikationslösung GUSbox ${ }^{\circledR}$ das Produktportfolio von dgnservice. (ЕВ) www.dgnservice.de

\section{Einfacheres Diktieren mit neuer Software}

Grundig Business Systems hat eine neue Version der Sprachverarbeitungssoftware DigtaSoft 4.7 entwickelt. Sie unterstützt die Version 11 der Spracherkennung Dragon Naturally Speaking und bietet mehr Sicherheit beim Versenden von Diktaten durch eine 256-Bit-Verschlüsselung. Verfügbar ist auch ein Update der dazugehörigen Software AdapterServer. (EB) www.grundig-gbs.com 